www.jmscr.igmpublication.org

Impact Factor 3.79

$\operatorname{ISSN}(\mathrm{e})-2347-176 \mathrm{x}$

crossref DOI: http://dx.doi.org/10.18535/jmscr/v3i8.56

Journal Of Medical Science And Clinical Research

\title{
Cardiac Sarcoidosis Causing Sudden Death
}

\author{
Authors \\ Gupta Kamal K. ${ }^{1}$, Behera Sameer K. ${ }^{2}$, Mishra Debi P. ${ }^{3}$ \\ ${ }^{1}$ Junior Resident, Department of Patholgy, M.K.C.G. Medical College, Berhampur, 760004, India \\ ${ }^{2}$ Assistant Professor, Department of Patholgy, M.K.C.G. Medical College, Berhampur, 760004, India \\ ${ }^{3}$ Professor and Head of Dept, Dept of Patholgy, M.K.C.G. Medical College, Berhampur, 760004, India \\ Corresponding Author
}

Dr. Kamal Kant Gupta

Junior Resident, Department of Pathology, M.KC.G. Medical College, Berhampur-764004, India

Email: Dr.kamalkantgupta@Gmail.com,Tel: 91-8093970832

\begin{abstract}
Sarcoidosis is a granulomatous disease of unclear etiology. Cardiac involvement in sarcoidosis is rarely diagnosed clinically. It is not commonly fatal, but when sarcoidosis is fatal, it is most often from cardiac involvement and when sarcoidosis involves the heart, it frequently causes death. We report a case of sudden death of 48 years male, wherein sarcoidosis with cardiac involvement was diagnosed at histopathological examination. We received a heart weighed $500 \mathrm{gms}$ with left ventricular wall thickness $3.5 \mathrm{~cm}$. On microscopic examination, the heart wall shows multiple noncaseous granuloma with few multinucleated giant cells and fibrosis around it. No acid fast bacilli identified on ziehlneelsen stain.
\end{abstract}

Keywords: Sarcoidosis, granulomatous, autopsy, sudden death

\section{INTRODUCTION}

Sarcoidosis is an aggressively studied systemic granulomatous disease that has been a source of considerable controversy due to unclear etiology and diverse presentation. Cardiac involvement in sarcoidosis is rarely diagnosed clinically. It is not commonly fatal, but when sarcoidosis is fatal, it is most often from cardiac involvement and when sarcoidosis involves the heart, it frequently causes death $^{[1]}$. The sarcoidosis usually diagnosed histologically with presence of granuloma without any necrosis and no acid fast bacill identified on ziehlneelsen stain. Granulomatous myocarditis is a term reserved for an entity in with well defined granulomas and should be differentiated from giant cell myocarditis (GCM). In cases of granuloma formation and multiorgan involvement, sarcoidosis and tuberculosis should be considered. The sarcoidosis especially when granuloma are not associated with necrosis [2]. We report a sudden death of male with granulomatous myocarditis and propose that cardiac sarcoidosis could have been the underlying etiology. Differential diagnosis for granulomatous myocarditis including sarcoidosis, tuberculosis as well as idiopathic giant cell myocrditis is discussed $^{[3]}$. 


\section{CASE REPORT}

A 48 years male suddenly collapsed at home. He was brought dead to the hospital. Enquiry revealed no significant symptoms or ill health before his death. We received heart from forensic department.

Gross lesions in the heart : Heart weighed 500gms, grossly increased in size and showed increased in epicardial fat. The left ventricular wall thickness is $3.5 \mathrm{~cm}$ (Figure 1). The left ventricular cavity narrowed. The other cardiac chambers, valves and coronaries were normal. Light microscopic examination revealed Multiple granuloma with fibrosis in the heart wall(Figure 2). The granulomatous lesions composed of lymphocytes and epithelioid cells in the myocardium accompanied with fibrosis without any obvious necrosis(Figure 3,4). Few multinucleated giant cells are scattered in these lesions. Serial sections through left ventricular wall revealed extensive granulomatous infiltration through the wall. Attempts to demonstrate acid fast bacilli to rule out tuberculosis by ziehlneelsen stain yielded negative results. A diagnosis of cardiac sarcoidosis was proposed, considering the nonnecrotizing granulomas.

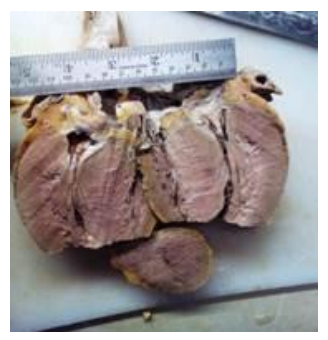

Figure 1: Gross apperance of heart, showing increased size of heart and thickened left ventricular wall.

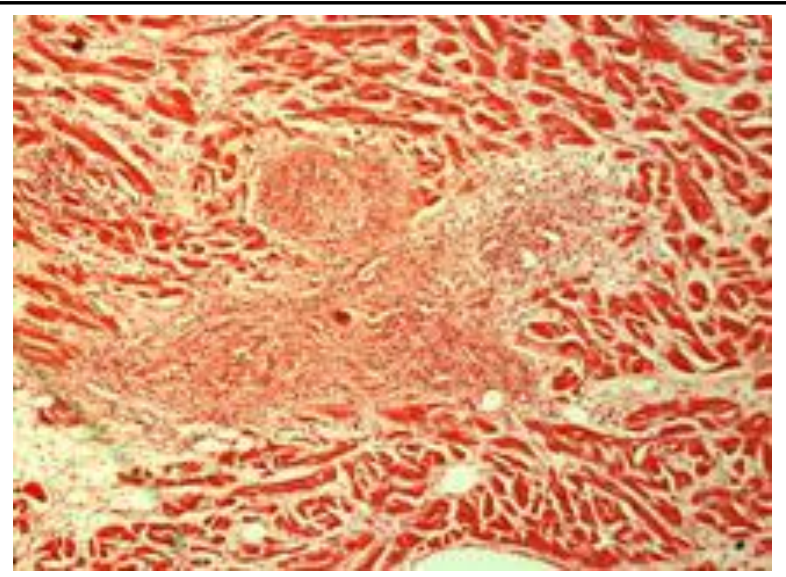

Figure 2: (Photomicrograph)Multiple granuloma with fibrosis in the heart wall [H and E, X40].

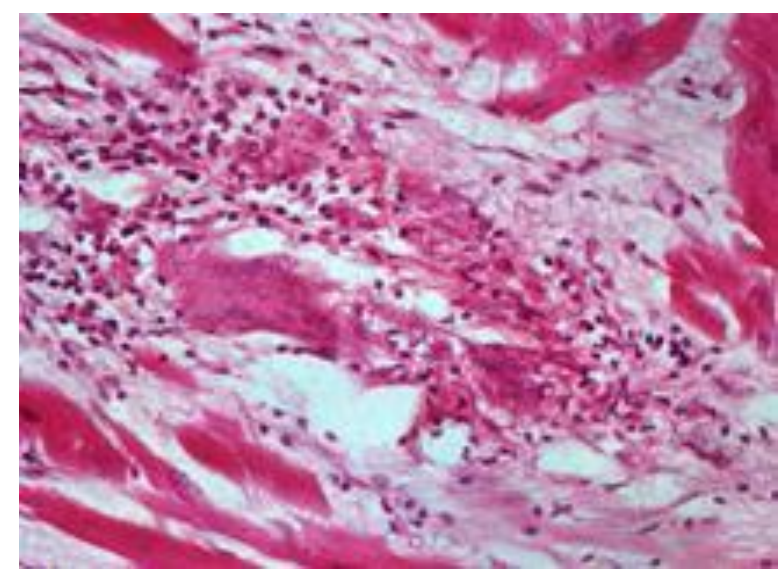

Figure 3: (Photomicrograph) Granuloma and fibrosis around it [H and E, X400].

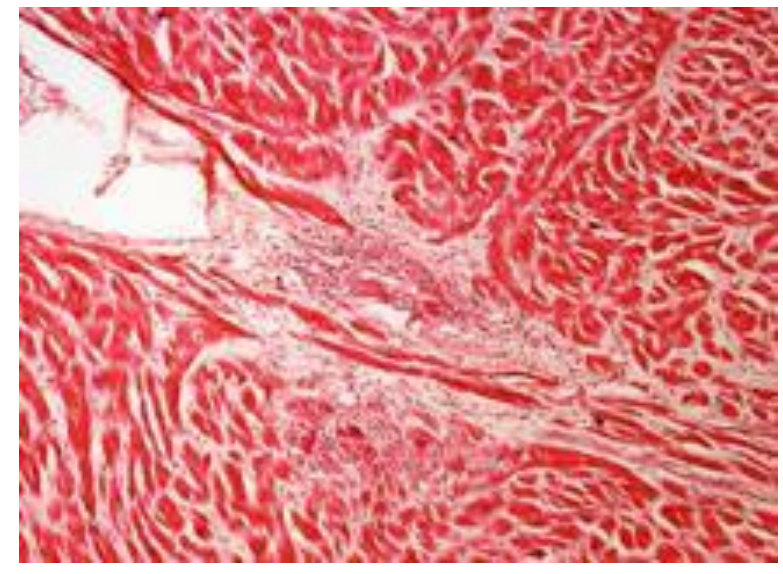

Figure 4: (photomicrograph) Granuloma consists of lymphocyte, epitheloid cells, few multinucleated giant cells without any necrosis $[\mathrm{H}$ and $\mathrm{E}$, $\mathrm{X} 400]$.

\section{DISCUSSION}

Sarcoidosis is a multisystemic granulomatous disease of unknown etiology ${ }^{[1]}$. The cardiovascular system is the third most frequently 
involved organ system in autopsy studies on patients with sarcoidosis following the lymphoid and respiratory systems ${ }^{[4]}$. Grossly the granulomatous lesions of cardiac sarcoidosis can appear as yellow, white, tan, light brown or gray irregular tumor like infiltrates ${ }^{[5]}$. In the heart, the myocardium of the left ventricular free wall is the most location of sarcoid granulomas, followed by the septum, right ventricle and atria ${ }^{[5]}$. The disease also commonly affects the cardiac conduction system $^{[6]}$.

The main differential diagnosis in a case of sudden death in a young patients with granulomatous myocarditis would be tuberculosis, sarcoidosis and giant cell myocarditis. Idiopathic GCM which is used to be classified with granulomatous lesion before is now thought to be a separate entity from granulomatous myocarditis due to lacks of true granuloma formation. Typical multifocal or widespread geographic necrosis , presence of myogenic giant cells, prominent eosinophilic infiltration and absence of extra cardiac granulomas or giant cells observed in GCM differentiate from sarcoidosis ${ }^{[7]}$. Our case lacked the above features making GCM an unlikely diagnosis. The immunohistochemical evidence suggest that giant cells of GCM are myogenic in origin, being derived from altered myocytes rather than tissue macrophages ${ }^{[8]}$. The characteristic giant cells of GCM are uniformly negative for cytoplasmic muramidase (CM) and positive for myoglobin whereas $\mathrm{CM}$ positive in tissue macrophages and giant cells of generalized sarcoidosis invades the heart ${ }^{[8]}$. On the basis of above findings suggest and support the concept of classifying GCM as a separate entity from sarcoidosis.

Tuberculosis, intially considered a close differential diagnosis was also unlikely because of absence of characteristic caseous necrosis in this case. Identification of mycobacterium by conventional stains is necessary to confirm the diagnosis. Stain was done in this case and no acid fast bacilli seen. Although there is no pathognomonic finding to differentiate a sarcoidal granuloma from a granuloma of another cause, some features favor a granuloma being sarcoidal. The granulomas of sarcoidosis are typically tight, naked, nonnecrotizing and epithelioid ${ }^{[9]}$. This criterion is not entirely reliable, however, as early sarcoidal granulomas are not tightly cohesive and may be have abundant lymphocytes. Overtime, sarcoidal granuloma become more compact and come to have less rim of lymphocytes ${ }^{[10]}$.

\section{CONCLUSION}

Sarcoidosis is a focal or disseminated granulomatous disease, which likely represents the final common pathway of various pathogenic insults in a genetically susceptible host. Although fairly rare, cardiac sarcoid is one of the possible menifestations that clinicians must be most vigilant about due to high risk of causing fatal arrythmias or congestive heart failure if untreated. Endomyocardial biopsy can make the diagnosis, but its sensitivity is limited by the patchy myocardial distribution of the granulomas.

To date, we do not know what predisposes some sarcoid patients to develop cardiac sarcoid. Genetic studies are beginning to provide answers to that question, but more work remians. An exciting idea is that the type of antigenic stimuli may influence the development of different sarcoid phenotypes, including cardiac sarcoid. Unfortunately, one of the ways cardiac sarcoidosis is commanly diagnosed still remains the autopsy, but this can great of great benefit for families and medical staff.

\section{CONFLICT OF INTEREST: NIL.}

\section{FUNDING: NIL.}

\section{REFERENCES}

1. Chapelon-Abric, Catherine, et al. "Cardiac sarcoidosis: a retrospective study of 41 cases." Medicine 83.6 (2004): 315-334

2. Suranagi, Vijayalaxmi V., Prakash R. Malur, and Hema B. Bannur. "Cardiac sarcoidosis causing sudden death." Indian 
Journal of Pathology and Microbiology 52.4 (2009): 566.

3. Mitchell, Richard N., Richard A. Jonas, and Frederick J. Schoen. "Pathology of explanted cryopreserved allograft heart valves: comparison with aortic valves from orthotopic heart transplants." The Journal of Thoracic and Cardiovascular Surgery 115.1 (1998): 118-127.

4. Perry, A., and F. Vuitch. "Causes of death in patients with sarcoidosis. A morphologic study of 38 autopsies with clinicopathologic correlations." Archives of pathology \& laboratory medicine 119.2 (1995): 167-172.

5. Roberts, William C., Hugh A. McAllister, and Victor J. Ferrans. "Sarcoidosis of the heart: a clinicopathologic study of 35 necropsy patients (group I) and review of 78 previously described necropsy patients (group II)." The American journal of medicine 63.1 (1977): 86-108.

6. Dubrey, Simon W., Alex Bell, and Tarun K. Mittal. "Sarcoid heart disease." Postgraduate medical journal 83.984 (2007): 618-623.

7. Cooper Jr, Leslie T., Gerald J. Berry, and Ralph Shabetai. "Idiopathic giant-cell myocarditis - natural history and treatment." New England Journal of Medicine336.26 (1997): 1860-1866.

8. Hamilton, Rebecca A., Linda Sullivan, and Barbara C. Wolf. "Sudden cardiac death due to giant cell inflammatory processes" Journal of forensic sciences52.4 (2007): 943-948.

9. Gal, Anthony A., and Michael N. Koss. "The pathology of sarcoidosis." Current opinion in pulmonary medicine 8.5 (2002): 445-451.

10. Rosen, Yale. "Pathology of sarcoidosis." Seminars in respiratory and critical care medicine. Vol. 28. No. 1. New
York: Thieme Medical Publishers, c1994-, 2007. 